\title{
Long-Term Weight Loss and Metabolic Syndrome Remission after Bariatric Surgery: The Effect of Sex, Age, Metabolic Parameters and Surgical Technique - A 4-Year Follow-Up Study
}

\author{
Vanessa Guerreiro ${ }^{a, b}$ João Sérgio Neves ${ }^{a, b}$ Daniela Salazar ${ }^{a, b}$ \\ Maria João Ferreira ${ }^{a, b}$ Sofia Castro Oliveira ${ }^{a, b}$ Pedro Souteiro ${ }^{a, b}$ \\ Jorge Pedro ${ }^{a, b}$ Daniela Magalhães ${ }^{a, b}$ Ana Varela ${ }^{a, b}$ Sandra Belo ${ }^{a, b}$ \\ Paula Freitas ${ }^{a-d}$ Davide Carvalho ${ }^{a, b} \quad$ AMTCO Group \\ aDepartment of Endocrinology, Diabetes and Metabolism, Centro Hospitalar São João, Porto, \\ Portugal; ${ }^{b}$ Faculty of Medicine, University of Porto, Porto, Portugal; ' Instituto de Investigação e

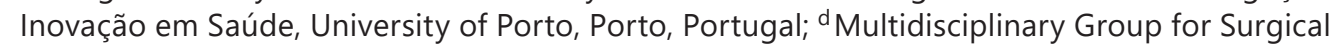 \\ Management of Obesity, Centro Hospitalar São João, Porto, Portugal
}

Keywords

Morbid obesity · Weight loss · Metabolic syndrome · Bariatric surgery

\begin{abstract}
Introduction: Bariatric surgery is an effective treatment for morbid obesity and its metabolic related comorbidities. However, the literature reports inconsistent results regarding weight loss (WL) and the resolution of comorbidities associated with obesity. Objective: We aim to evaluate long-term differences in WL between different surgical techniques and the impact of each surgical technique on metabolic parameters (type 2 diabetes mellitus [T2DM], dyslipidemia, hypertension, and metabolic syndrome). We also aim to evaluate the effect of baseline clinical characteristics in WL and in the evolution of metabolic syndrome (MetS) components. Our hypothesis is that different types of surgery have different effects on WL and the prevalence of comorbidities over time. Methods: We retrospectively evaluated WL and metabolic parameter remission (T2DM, dyslipidemia, hypertension, and MetS) during 4 years in 1,837 morbidly obese patients (females, $85 \%$; age, $42.5 \pm 10.6$ years; BMI, $44.0 \pm 5.8$ ) who underwent bariatric surgery (Roux-en-Y gastric bypass [RYGB], laparoscopic sleeve gastrectomy [LSG], and laparoscopic adjustable gastric band [LAGB]). Results: The mean percentage of WL for RYGB, LSG, and LAGB was, respectively, $32.9 \pm 8.7,29.8 \pm 9.8$, and $16.2 \pm 9.6$ at 12 months and $30.6 \pm 9.1,22.7 \pm 10.0$, and $15.8 \pm 10.8$ at 48 months $(p<0.001)$, even after adjustment for baseline weight, $\mathrm{BMI}$, age, and sex $(p<0.001)$. Women had more WL during the first 36
\end{abstract}


months ( $p=0.013$ and 0.007 at 12 and 36 months, respectively) and older patients had less WL compared to younger ones ( $p<0.001$ ), except at 48 months. Patients with T2DM had less $W L$ than those without diabetes after adjustment (sex, age, and surgical technique) during the same period. Patients with hypertension had less WL at 12 months $(p=0.009)$ and MetS at 24 months ( $p=0.020$ ) compared to those without these comorbidities. There was no significant difference regarding the presence of dyslipidemia in WL. The RYGB group showed better results for MetS resolution. Conclusion: During the 4-year follow-up, RYGB was the surgical procedure that caused the highest WL and MetS resolution.

(C) 2019 The Author(s)

Published by S. Karger AG, Basel

\section{Introduction}

Obesity is an increasing health problem worldwide, the consequences of which are well recognized and include several comorbidities such as hypertension, type 2 diabetes mellitus (T2DM), and dyslipidemia.

Treatment options for obesity include lifestyle modifications, medications, and bariatric surgery (BS), with the last one being the most effective treatment [10].

While BS is highly effective, the literature is scarce and there is controversy regarding the effects of each surgical technique on long-term weight loss (WL) after BS $[3,12,14,15]$.

It is well known that BS also decreases the prevalence of obesity-related comorbidities, e.g., the improvement and remission of T2DM in individuals with obesity are substantially better after BS than after conventional medical therapy [20], although the differential effect of RYGB, LSG, and LAGB on the long-term remission of metabolic syndrome (MetS) in unclear [21].

We aim to evaluate long-term differences in WL between different surgical techniques and the impact of each surgical technique on metabolic parameters (diabetes, dyslipidemia, hypertension, and MetS). We also aim to evaluate the effect of baseline clinical characteristics in WL and in the evolution of MetS components. Our hypothesis is that different types of surgery have different effects on WL and the prevalence of comorbidities over time.

\section{Methods}

\section{Study Design and Participants}

We carried out a retrospective observational study on all morbidly obese patients submitted to BS - RYGB, LAGB, or LSG - between January 2010 and June 2016 in our Center. We evaluated the effect of BS in WL and on the evolution of metabolic parameters (diabetes, dyslipidemia, hypertension, and MetS). Data were collected from visits that occurred preoperatively and annually postoperatively during 4 years.

A total of 1,837 patients underwent BS during this time. All of the study participants had either a BMI $>40$ or an obesity-related comorbidity and a BMI $>35$, and all complied with a dietary plan for at least 12 months.

\section{Clinical Parameters Evaluated}

The following preoperative parameters were collected: age; sex; weight; BMI; waist circumference; hip circumferences (HC); blood pressure (BP); history of dyslipidemia, diabetes or hypertension; and type of BS performed (LAGB, RYGB, or LSG). WL and comorbidity remission over 4 years after the surgery were also evaluated.

We defined BMI as an individual's body weight $(\mathrm{kg})$ divided by the square of their height $(\mathrm{m})$. Excess WL (EWL) as a percentage during 4 years after the surgery was evaluated using 
Table 1. Patients' baseline characteristics

\begin{tabular}{lc}
\hline Females & 85.0 \\
Age, years & $42.5 \pm 10.6$ \\
BMI & $44.0 \pm 5.8$ \\
Weight, kg & $116.1 \pm 18.9$ \\
Abdominal circumference, cm & $123.3 \pm 13.4$ \\
Hip circumference, cm & $132.4 \pm 11.6$ \\
Diabetes & 29.7 \\
Dyslipidemia & 42.9 \\
Hypertension & 58.4 \\
Metabolic syndrome & 64.6 \\
SBP, mm Hg & $133.2 \pm 17.4$ \\
DBP, mm Hg & $83.1 \pm 11.2$ \\
LDL, mg/dL & $124.6 \pm 33.7$ \\
HDL, mg/dL & $49.7 \pm 11.3$ \\
TG, mg/dL & $139.6 \pm 80.9$ \\
Glycemia, mg/dL & $102.4 \pm 36.2$ \\
A1c & $5.9 \pm 1.1$ \\
\hline
\end{tabular}

Values are presented as means \pm SD or percents. The total number of patients is 1,837. A1c, glycated hemoglobin.

the following formula: ([preoperative weight - current weight]/[preoperative weight - ideal weight to produce a BMI of 25]) $\times 100$.

To define T2DM, we used the 2019 ADA guideline criteria, i.e., HbA1c $\geq 6.5 \%$, fasting plasma glucose of $126 \mathrm{mg} / \mathrm{dL}$, or 2 -h postload plasmatic glucose $\geq 200 \mathrm{mg} / \mathrm{dL}$ during an OGTT. We also considered patients to have diabetes if they were under antidiabetic treatment. We deemed patients to be in diabetes remission if they had diabetes in year 0 and, during follow-up, did not meet any of the 2019 ADA guidelines criteria and were not under antidiabetic treatment. Hypertension was defined as a systolic BP (SBP) $\geq 140$ or a diastolic BP (DBP) $\geq 90 \mathrm{~mm} \mathrm{Hg}$, or undergoing antihypertensive therapy. Dyslipidemia was defined as serum low-density lipoprotein (LDL) cholesterol $\geq 160 \mathrm{mg} / \mathrm{dL}$, serum high-density lipoprotein (HDL) cholesterol $<40 \mathrm{mg} / \mathrm{dL}$, or serum triglycerides $\geq 200 \mathrm{mg} / \mathrm{dL}$, or being on lipid-lowering agents.

MetS was defined as the presence of central obesity (WC $\geq 94 \mathrm{~cm}$ in men or $\geq 80 \mathrm{~cm}$ in women) and at least 2 of the following criteria: fasting glucose $\geq 100 \mathrm{mg} / \mathrm{dL}(5.6 \mathrm{mmol} / \mathrm{L})$ and/or T2DM; SBP $\geq 130 \mathrm{~mm} \mathrm{Hg}$, PAD $\geq 85 \mathrm{~mm} \mathrm{Hg}$, and/or specific treatment; triglycerides $\geq 150 \mathrm{mg} / \mathrm{dL}(1.7 \mathrm{mmol} / \mathrm{L})$ and/or specific treatment; and HDL-C levels < $40 \mathrm{mg} / \mathrm{dL}$ (1 $\mathrm{mmol} / \mathrm{L})$ in men or $<50 \mathrm{mg} / \mathrm{dL}(1.3 \mathrm{mmol} / \mathrm{L})$ in women and/or specific treatment, using International Diabetes Federation criteria.

\section{Statistical Analysis}

For comparison of baseline characteristics and variations in clinical and laboratory parameters according to the type of surgery, we carried out linear regression models and used logistic regression models which were unadjusted and adjusted for sex and age. For comparison of EWL and BMI after surgery, according to age group, sex, presence of diabetes, hypertension, dyslipidemia, and MetS, we used linear regression models which were unadjusted and adjusted for sex, age, and type of surgery. Results are presented as means \pm SD for continuous variables and as percents for categorical variables. Statistical analyses were carried out using Stata software, version 14.1 (StataCorp). Two-sided $p<0.05$ was considered statistically significant. The datasets used and/or analyzed during the current study are available from the corresponding author upon reasonable request. 
Guerreiro et al.: WL MetS Remission after BS

Table 2. Patients' baseline characteristics by type of surgery

\begin{tabular}{|c|c|c|c|c|}
\hline & RYBG & LSG & LAGB & $p$ \\
\hline Total & $1,100(59.88)$ & 466 (25.37) & $271(14.75)$ & \\
\hline $\begin{array}{l}\text { Female } \\
p \text { (adjusted) } \\
p \text { (not adjusted) }\end{array}$ & 960 (87.27) & 369 (79.18) & $232(85.61)$ & $\begin{array}{l}<0.001 \\
<0.001\end{array}$ \\
\hline $\begin{array}{l}\text { Age, years } \\
p \text { (adjusted) } \\
p \text { (not adjusted) }\end{array}$ & $41.60 \pm 10.12$ & $43.53 \pm 11.60$ & $44.63 \pm 10.58$ & $\begin{array}{l}<0.001 \\
<0.001\end{array}$ \\
\hline $\begin{array}{l}\text { BMI } \\
p \text { (adjusted) } \\
p \text { (not adjusted) }\end{array}$ & $44.25 \pm 5.52$ & $43.91 \pm 6.52$ & $43.25 \pm 5.30$ & $\begin{array}{l}0.031 \\
0.036\end{array}$ \\
\hline $\begin{array}{l}\text { Weight, } \mathrm{kg} \\
p \text { (adjusted) } \\
p \text { (not adjusted) }\end{array}$ & $116.77 \pm 18.11$ & $117.30 \pm 21.47$ & $111.35 \pm 16.44$ & $\begin{array}{l}<0.001 \\
<0.001\end{array}$ \\
\hline $\begin{array}{l}\text { Waist circumference, } \mathrm{cm} \\
p \text { (adjusted) } \\
p \text { (not adjusted) }\end{array}$ & $123.19 \pm 13.24$ & $123.67 \pm 14.55$ & $122.86 \pm 12.01$ & $\begin{array}{l}0.561 \\
0.764 \\
\end{array}$ \\
\hline $\begin{array}{l}\text { Hip circumference, } \mathrm{cm} \\
p \text { (adjusted) } \\
p \text { (not adjusted) }\end{array}$ & $133.13 \pm 11.77$ & $131.43 \pm 11.65$ & $130.83 \pm 10.66$ & $\begin{array}{l}0.088 \\
0.010\end{array}$ \\
\hline $\begin{array}{l}\text { Diabetes } \\
p \text { (adjusted) } \\
p \text { (not adjusted) }\end{array}$ & $519(28.25)$ & $557(30.20)$ & 637 (34.69) & $\begin{array}{l}0.503 \\
0.113\end{array}$ \\
\hline $\begin{array}{l}\text { Dyslipidemia } \\
p \text { (adjusted) } \\
p \text { (not adjusted) }\end{array}$ & $740(40.26)$ & 837 (45.60) & 902 (49.08) & $\begin{array}{l}0.233 \\
0.013\end{array}$ \\
\hline $\begin{array}{l}\text { Hypertension } \\
p \text { (adjusted) } \\
p \text { (not adjusted) }\end{array}$ & $1,050(57.16)$ & $1,034(56.29)$ & $1,225(66.67)$ & $\begin{array}{r}<0.001 \\
0.012\end{array}$ \\
\hline $\begin{array}{l}\text { Metabolic syndrome } \\
p \text { (adjusted) } \\
p \text { (not adjusted) }\end{array}$ & $1,175(63.95)$ & $1,271(69.21)$ & $114(60.62)$ & $\begin{array}{l}0.050 \\
0.105\end{array}$ \\
\hline $\begin{array}{l}\mathrm{A} 1 \mathrm{c}, \% \\
p \text { (adjusted) } \\
p \text { (not adjusted) }\end{array}$ & $5.89 \pm 1.13$ & $5.82 \pm 0.95$ & $5.99 \pm 1.08$ & $\begin{array}{r}<0.001 \\
0.109\end{array}$ \\
\hline
\end{tabular}

Values are presented as numbers $(\%)$ or means \pm SD. A1c, glycated hemoglobin.

\section{Results}

Baseline Population Characteristics

Of the 1,837 patients under observation $(59.9 \%$ submitted to RYGB, $25.4 \%$ to LSG, and $14.4 \%$ to LAGB), $85 \%$ were women with an average age of $42.5 \pm 10.6$ years (Table 1 ). The mean initial BMI was $44.0 \pm 5.8$, the mean WC was $123.3 \pm 13.4 \mathrm{~cm}$, and the mean HC was $132.4 \pm 11.6 \mathrm{~cm} ; 29.7 \%$ of the patients had T2DM, $42.9 \%$ had dyslipidemia, $58.4 \%$ had hypertension, and $64.8 \%$ had MetS.

The female sex was predominant in all 3 groups but mainly in the RYGB group $(p<0.001)$ (Table 2). The patients in the RYGB group were younger $(p=0.036)$ and they were the heaviest at baseline $(p<0.001)$, with a mean preoperative BMI of 44.3 for the RYGB group. For RYGB, 
Table 3. Weight change from baseline

\begin{tabular}{lllll}
\hline & Year 1 & Year 2 & Year 3 & Year 4 \\
\hline Total & $n=1,665$ & $n=1,092$ & $n=754$ & $n=473$ \\
\hline Weight loss, kg & $34.8 \pm 15.0$ & $35.1 \pm 15.9$ & $31.9 \pm 15.9$ & $30.1 \pm 15.2$ \\
BMI decrease & $13.2 \pm 5.4$ & $13.4 \pm 5.9$ & $12.3 \pm 6.0$ & $11.6 \pm 5.7$ \\
Weight loss, \% & $29.7 \pm 10.8$ & $29.8 \pm 11.6$ & $27.4 \pm 12.1$ & $15.9 \pm 11.6$ \\
EWL, \% & $71.1 \pm 26.8$ & $70.4 \pm 27.6$ & $65.0 \pm 29.1$ & $61.4 \pm 27.5$ \\
\hline RYGB & $n=1003$ & $n=645$ & $n=466$ & $n=296$ \\
Weight loss, kg & $38.6 \pm 13.2^{\mathrm{a}, \mathrm{b}}$ & $40.1 \pm 13.5^{\mathrm{a}, \mathrm{b}}$ & $38.0 \pm 13.6^{\mathrm{a}}, \mathrm{b}$ & $35.8 \pm 13.1^{\mathrm{a}, \mathrm{b}}$ \\
BMI decrease & $14.6 \pm 5.1^{\mathrm{a}, \mathrm{b}}$ & $15.3 \pm 5.0^{\mathrm{a}, \mathrm{b}}$ & $14.5 \pm 5.1^{\mathrm{a}, \mathrm{b}}$ & $13.7 \pm 4.8^{\mathrm{a}, \mathrm{b}}$ \\
Weight loss, \% & $32.9 \pm 8.7^{\mathrm{a}, \mathrm{b}}$ & $34.1 \pm 9.0^{\mathrm{a}, \mathrm{b}}$ & $32.4 \pm 9.4^{\mathrm{a}, \mathrm{b}}$ & $30.6 \pm 9.1^{\mathrm{a}, \mathrm{b}}$ \\
EWL, \% & $78.4 \pm 22.2^{\mathrm{a}, \mathrm{b}}$ & $80.1 \pm 22.0^{\mathrm{a}, \mathrm{b}}$ & $76.6 \pm 22.9^{\mathrm{a}, \mathrm{b}}$ & $72.3 \pm 22.1^{\mathrm{a}, \mathrm{b}}$ \\
\hline LSG & $n=415$ & $n=258$ & $n=122$ & $n=60$ \\
Weight loss, kg & $35.3 \pm 14.3^{\mathrm{a}}$ & $34.4 \pm 15.4^{\mathrm{a}}$ & $28.3 \pm 14.4^{\mathrm{a}}$ & $26.6 \pm 13.1^{\mathrm{a}}$ \\
BMI decrease & $13.1 \pm 5.0^{\mathrm{a}}$ & $13.0 \pm 5.6^{\mathrm{a}}$ & $10.8 \pm 5.3^{\mathrm{a}}$ & $10.3 \pm 5.0^{\mathrm{a}}$ \\
Weight loss, \% & $29.8 \pm 9.8^{\mathrm{a}}$ & $28.7 \pm 10.8^{\mathrm{a}}$ & $24.3 \pm 11.3^{\mathrm{a}}$ & $22.7 \pm 10.0^{\mathrm{a}}$ \\
EWL, \% & $72.8 \pm 25.3^{\mathrm{a}}$ & $68.0 \pm 26.1^{\mathrm{a}}$ & $57.5 \pm 27.7^{\mathrm{a}}$ & $53.3 \pm 23.8^{\mathrm{a}}$ \\
\hline LABG & $n=247$ & $n=189$ & $n=166$ & $n=117$ \\
Weight loss, kg & $18.5 \pm 11.8$ & $18.8 \pm 12.8$ & $17.5 \pm 12.7$ & $17.7 \pm 13.2$ \\
BMI decrease & $7.2 \pm 4.5$ & $7.3 \pm 4.9$ & $6.9 \pm 5.0$ & $6.9 \pm 5.1$ \\
Weight loss, \% & $16.2 \pm 9.6$ & $16.8 \pm 10.4$ & $15.6 \pm 10.5$ & $15.7 \pm 10.8$ \\
EWL, \% & $39.2 \pm 23.2$ & $40.7 \pm 24.7$ & $38.0 \pm 25.7$ & $38.0 \pm 26.0$ \\
\hline
\end{tabular}

a Significant difference $(p<0.05)$ vs. a band, even after adjustment for baseline weight, BMI, age, and sex. b Significant difference $(p<0.05)$ vs. LSG, even after adjustment for baseline weight, BMI, age, and sex.

28.3\% had T2DM, 57.2\% had hypertension, 40.3\% had dyslipidemia, and 64.0\% had MetS. The corresponding presence of comorbidities in LSG at baseline was 30.2, 56.3, 45.6, and $69.2 \%$ and in LAGB it was 34.7, 49.1, and 66.6\%, respectively. Hypertension was significantly higher in the LAGB group $(p<0.001)$.

The follow-up compliance rates at 12, 24, 36, and 48 months were: $91.2,58.6,42.4$, and $26.9 \%$ for the RYGB group; 89.1, 55.4, 26.2, and 12.9\% for the LSG group; and 91.1, 69.7, 61.2, and $43.2 \%$ for the LAGB group, respectively. Among the initial population, $90.1 \%$ of the patients had 1 year of follow-up, $59.4 \%$ had 2 years, $41.0 \%$ had 3 years, and $25.7 \%(n=473)$ had 4 years.

\section{Impact on $W L$}

One year after the surgery, patients undergoing RYGB lost $38.6 \pm 13.2 \mathrm{~kg}$ of total weight, $32.9 \pm 8.7 \%$ of total weight, and $78.4 \pm 22.2 \%$ of excess weight, while patients in the LSG group lost $35.3 \pm 14.3 \mathrm{~kg}, 29.8 \pm 9.8 \%$ of total weight, and $72.8 \pm 25.3 \%$ of excess weight, respectively, and patients in the LAGB group lost $18.5 \pm 11.8 \mathrm{~kg}, 16.2 \pm 9.6 \%$ of total weight, and 39.2 $\pm 23.2 \%$ of excess weight $(p<0.001$ ) (Table 3 ). The differences were preserved in the subsequent years: the mean percent of total WL (TWL) for RYGB, LSG, and LAGB was $30.6 \pm 9.1$, $22.7 \pm 10.0$, and $15.8 \pm 10.8$ at 48 months, respectively $(p<0.001)$. The mean percent of EWL for RYGB, LSG, and LAGB was, respectively, $72.3 \pm 22.1,53.3 \pm 23.8$, and $38.0 \pm 26.0$ at 48 months $(p<0.001)$.

Older patients had less WL $(p<0.001)$, except at 48 months (Table 4$)$, when the difference was not statistically significant ( $p=0.102)$. For example, during the first year, patients older 
Table 4. EWL by baseline characteristics

\begin{tabular}{|c|c|c|c|c|}
\hline & Year 1 & Year 2 & Year 3 & Year 4 \\
\hline Total & $n=1,665$ & $n=1,092$ & $n=754$ & $n=473$ \\
\hline \multicolumn{5}{|l|}{ Age, years } \\
\hline$<40$ & $76.5 \pm 26.0$ & $76.1 \pm 26.8$ & $70.0 \pm 29.7$ & $65.9 \pm 28.9$ \\
\hline $40-50$ & $71.4 \pm 26.0$ & $70.6 \pm 27.4$ & $65.9 \pm 28.6$ & $60.3 \pm 24.2$ \\
\hline$>50$ & $62.1 \pm 26.7$ & $61.5 \pm 26.7$ & $56.9 \pm 27.0$ & $56.6 \pm 27.8$ \\
\hline$p$ (not adjusted) & $<0.001$ & $<0.001$ & $<0.001$ & 0.007 \\
\hline$p$ (adjusted) & $<0.001$ & $<0.001$ & $<0.001$ & 0.102 \\
\hline \multicolumn{5}{|l|}{ Gender } \\
\hline Male & $66.7 \pm 24.9$ & $66.0 \pm 23.6$ & $57.6 \pm 28.0$ & $56.9 \pm 24.9$ \\
\hline Female & $71.9 \pm 27.0$ & $71.0 \pm 28.1$ & $65.9 \pm 29.1$ & $62.0 \pm 27.9$ \\
\hline$p$ (not adjusted) & 0.005 & 0.048 & 0.013 & 0.186 \\
\hline$p$ (adjusted) & 0.013 & 0.030 & 0.010 & 0.391 \\
\hline \multicolumn{5}{|l|}{ Diabetes $^{\mathrm{a}}$} \\
\hline Yes & $65.9 \pm 24.6$ & $63.9 \pm 25.8$ & $59.3 \pm 26.7$ & $57.5 \pm 25.8$ \\
\hline No & $73.5 \pm 27.4$ & $73.2 \pm 27.9$ & $67.9 \pm 29.9$ & $63.4 \pm 28.2$ \\
\hline$p$ (not adjusted) & $<0.001$ & $<0.001$ & $<0.001$ & 0.027 \\
\hline$p$ (adjusted) & 0.031 & 0.001 & 0.007 & 0.124 \\
\hline \multicolumn{5}{|l|}{ Hypertension $^{\mathrm{a}}$} \\
\hline Yes & $67.9 \pm 26.3$ & $67.6 \pm 26.7$ & $63.2 \pm 27.8$ & $60.6 \pm 26.9$ \\
\hline No & $76.2 \pm 26.6$ & $75.0 \pm 27.5$ & $68.5 \pm 30.3$ & $62.6 \pm 28.6$ \\
\hline$p$ (not adjusted) & $<0.001$ & $<0.001$ & 0.015 & 0.425 \\
\hline$p$ (adjusted) & 0.009 & 0.171 & 0.718 & 0.907 \\
\hline \multicolumn{5}{|l|}{ Dyslipidemia $^{\mathrm{a}}$} \\
\hline Yes & $68.1 \pm 28.1$ & $66.4 \pm 27.7$ & $61.7 \pm 29.7$ & $61.2 \pm 28.9$ \\
\hline No & $73.5 \pm 25.5$ & $73.1 \pm 27.0$ & $67.3 \pm 28.2$ & $61.7 \pm 26.4$ \\
\hline$p$ (not adjusted) & $<0.001$ & $<0.001$ & 0.009 & 0.850 \\
\hline$p$ (adjusted) & 0.263 & 0.073 & 0.385 & 0.239 \\
\hline \multicolumn{5}{|l|}{$M e t S^{\mathrm{a}}$} \\
\hline Yes & $69.6 \pm 26.0$ & $67.7 \pm 26.0$ & $62.9 \pm 28.4$ & $60.8 \pm 26.3$ \\
\hline No & $72.9 \pm 28.4$ & $72.9 \pm 29.0$ & $65.8 \pm 30.1$ & $62.8 \pm 27.8$ \\
\hline$p$ (not adjusted) & 0.038 & 0.008 & 0.264 & 0.488 \\
\hline$p$ (adjusted) & 0.202 & 0.020 & 0.141 & 0.274 \\
\hline
\end{tabular}

Values are percents and they are presented as means \pm SD. ${ }^{a}$ Diabetes if $\mathrm{HbA} 1 \mathrm{c} \geq 6.5 \%$, fasting plasma glucose $=126 \mathrm{mg} / \mathrm{dL}$, or 2 -h postload plasmatic glucose $\geq 200 \mathrm{mg} / \mathrm{dL}$ during OGTT prior to bariatric surgery. Adjusted for age, gender, type of surgery (sex and age adjusted only for other variables).

than 50 years lost $61.1 \pm 26.7 \%$ of EWL versus $71.4 \pm 26.0 \%$ of EWL for those aged between 40 and 50 years versus $76.5 \pm 26.0 \%$ of EWL in those younger than 40 years. Women had more WL during the first 36 months $(p=0.013,0.001$, and 0.007 at 12,24 , and 36 months respectively). Patients with T2DM had less WL than those without T2DM after adjustment (for sex, age, and surgical technique) during the first 36 months ( $65.9 \pm 24.6$ vs. $73.5 \pm 27.4$, $63.9 \pm 25.8$ vs. $73.2 \pm 27.9$, and $59.3 \pm 26.7$ vs. $67.9 \pm 29.9 ; p=0.031,0.001$, and 0.007 at 12 , 24 , and 36 months, respectively), as did those with hypertension at 12 months ( $p=0.009)$ and those with MetS at 24 months $(p=0.020)$, compared to those without these comorbidities. There was no statistically significant difference in the presence of dyslipidemia. Patients with remission of T2DM during follow-up had a greater WL (adjusted analysis: difference 
Table 5. BMI variation by baseline characteristics

\begin{tabular}{|c|c|c|c|c|}
\hline & Year 1 & Year 2 & Year 3 & Year 4 \\
\hline Total & $n=1,655$ & $n=1,092$ & $n=754$ & $n=473$ \\
\hline \multicolumn{5}{|l|}{ Age, years } \\
\hline$<40$ & $14.2 \pm 5.2$ & $14.5 \pm 5.8$ & $13.2 \pm 6.2$ & $12.4 \pm 6.1$ \\
\hline $40-50$ & $13.0 \pm 5.2$ & $13.3 \pm 5.8$ & $12.4 \pm 5.8$ & $11.2 \pm 5.1$ \\
\hline$>50$ & $11.5 \pm 5.4$ & $11.8 \pm 5.8$ & $10.8 \pm 5.6$ & $10.8 \pm 5.6$ \\
\hline$p$ (not adjusted) & $<0.001$ & $<0.001$ & $<0.001$ & $<0.001$ \\
\hline$p$ (adjusted) & $<0.001$ & $<0.001$ & 0.007 & $<0.001$ \\
\hline \multicolumn{5}{|l|}{ Gender } \\
\hline Male & $13.1 \pm 5.9$ & $13.4 \pm 6.0$ & $11.3 \pm 6.1$ & $11.0 \pm 5.6$ \\
\hline Female & $13.6 \pm 5.3$ & $13.4 \pm 5.9$ & $12.4 \pm 6.0$ & $11.7 \pm 5.7$ \\
\hline$p$ (not adjusted) & 0.975 & 0.971 & 0.105 & 0.427 \\
\hline$p$ (adjusted) & 0.358 & 0.905 & 0.125 & 0.786 \\
\hline \multicolumn{5}{|l|}{ Diabetes $^{\mathrm{a}}$} \\
\hline Yes & $12.6 \pm 5.5$ & $12.6 \pm 6.1$ & $11.4 \pm 5.6$ & $10.8 \pm 5.1$ \\
\hline No & $13.4 \pm 5.3$ & $13.7 \pm 5.8$ & $12.7 \pm 6.1$ & $12.0 \pm 5.9$ \\
\hline$p$ (not adjusted) & 0.012 & 0.003 & 0.004 & 0.028 \\
\hline$p$ (adjusted) & 0.331 & 0.340 & 0.098 & 0.117 \\
\hline \multicolumn{5}{|l|}{ Hypertension $^{\mathrm{a}}$} \\
\hline Yes & $13.1 \pm 5.6$ & $13.2 \pm 5.9$ & $12.1 \pm 5.8$ & $11.7 \pm 5.7$ \\
\hline No & $13.3 \pm 5.1$ & $13.7 \pm 5.7$ & $12.5 \pm 6.2$ & $11.4 \pm 5.6$ \\
\hline$p$ (not adjusted) & 0.327 & 0.232 & 0.395 & 0.684 \\
\hline$p$ (adjusted) & 0.001 & 0.030 & 0.151 & 0.124 \\
\hline \multicolumn{5}{|l|}{ Dyslipidemia $^{\mathrm{a}}$} \\
\hline Yes & $12.6 \pm 5.4$ & $12.6 \pm 5.8$ & $11.4 \pm 5.8$ & $11.4 \pm 5.8$ \\
\hline No & $13.5 \pm 5.3$ & $13.9 \pm 5.9$ & $12.9 \pm 6.1$ & $11.8 \pm 5.6$ \\
\hline$p$ (not adjusted) & $<0.001$ & 0.001 & 0.001 & 0.444 \\
\hline$p$ (adjusted) & 0.248 & 0.106 & 0.028 & 0.792 \\
\hline \multicolumn{5}{|l|}{$M e t S^{\mathrm{a}}$} \\
\hline Yes & $13.3 \pm 5.4$ & $13.3 \pm 5.9$ & $12.2 \pm 5.9$ & $11.6 \pm 5.7$ \\
\hline No & $13.0 \pm 5.4$ & $13.2 \pm 5.9$ & $12.0 \pm 6.3$ & $11.7 \pm 5.7$ \\
\hline$p$ (not adjusted) & 0.352 & 0.844 & 0.758 & 0.949 \\
\hline$p$ (adjusted) & 0.024 & 0.332 & 0.898 & 0.780 \\
\hline
\end{tabular}

between patients with diabetes remission vs. without remission: year 1: $4.3 \pm 1.1, p<0.05$; year 2: $3.9 \pm 1.3, p=0.004$; year $3: 3.7 \pm 1.5, p=0.013$; and year $4: 2.4 \pm 1.7, p=0.167$ ) and BMI decrease (adjusted analysis: difference between patients with diabetes remission vs. without remission: year 1: $1.4 \pm 0.4, p<0.05$; year 2: $1.5 \pm 0.5, p=0.003$; year 3: $1.5 \pm 0.5, p=$ 0.007 , and year $4: 1.0 \pm 0.6, p=0.123$ ) during the first 3 years of follow-up. The differences in WL between patients with and without T2DM were also significant when analyzed by least square differences (year 1: -2.4 [ -4.0 to -0.8 ], year 2: -3.7 [ -5.8 to -1.7$]$, year 3: -4.4 [ -6.8 to $-2.0]$, and year 4: -3.7 [-6.6 to -0.8]). During the follow-up, there was a significant association between BMI loss and age. The presence of dyslipidemia also influenced BMI loss but only at 36 months (Table 5). 
Table 6. Metabolic changes from baseline

\begin{tabular}{|c|c|c|c|c|}
\hline & Year 1 & Year 2 & Year 3 & Year 4 \\
\hline Total & $n=1,655$ & $n=1,092$ & $n=754$ & $n=473$ \\
\hline \multicolumn{5}{|c|}{ Abdominal circumference, $\mathrm{cm}$} \\
\hline LABG & $-17.7 \pm 12.4$ & $-18.4 \pm 13.2$ & $-14.4 \pm 13.9$ & $-13.8 \pm 12.1$ \\
\hline LSG & $-27.2 \pm 12.2$ & $-25.5 \pm 14.0$ & $-21.2 \pm 14.9$ & $-25.8 \pm 16.7$ \\
\hline RYGB & $-29.2 \pm 11.6$ & $-32.7 \pm 12.2$ & $-29.4 \pm 11.2$ & $-28.7 \pm 14.0$ \\
\hline$p$ (not adjusted) & $<0.001$ & $<0.001$ & $<0.001$ & $<0.001$ \\
\hline$p$ (adjusted) & $<0.001$ & $<0.001$ & $<0.001$ & $<0.001$ \\
\hline \multicolumn{5}{|c|}{ Hip circumference, $\mathrm{cm}$} \\
\hline LABG & $-13.5 \pm 9.3$ & $-12.5 \pm 9.9$ & $-10.5 \pm 9.0$ & $-10.2 \pm 11.0$ \\
\hline LSG & $-22.5 \pm 10.3$ & $-22.3 \pm 12.6$ & $-19.5 \pm 10.1$ & $-19.6 \pm 10.1$ \\
\hline RYGB & $-26.4 \pm 11.1$ & $-28.6 \pm 11.5$ & $-25.5 \pm 11.5$ & $-24.9 \pm 12.6$ \\
\hline$p$ (not adjusted) & $<0.001$ & $<0.001$ & $<0.001$ & $<0.001$ \\
\hline$p$ (adjusted) & $<0.001$ & $<0.001$ & $<0.001$ & $<0.001$ \\
\hline \multicolumn{5}{|l|}{$S B P$} \\
\hline LABG & $-8.2 \pm 21.2$ & $-9.7 \pm 21.9$ & $-8.8 \pm 22.6$ & $-9.4 \pm 19.7$ \\
\hline LSG & $-8.7 \pm 18.7$ & $-10.6 \pm 19.5$ & $-4.4 \pm 20.0$ & $-11.5 \pm 22.1$ \\
\hline RYGB & $-13.2 \pm 20.4$ & $-11.0 \pm 20.8$ & $-8.8 \pm 22.0$ & $-11.2 \pm 21.8$ \\
\hline$p$ (not adjusted) & 0.004 & 0.855 & 0.396 & 0.870 \\
\hline$p$ (adjusted) & 0.037 & 0.998 & 0.704 & 0.692 \\
\hline \multicolumn{5}{|l|}{$D B P$} \\
\hline LABG & $-3.2 \pm 14.6$ & $-3.9 \pm 14.5$ & $-5.0 \pm 15.1$ & $-5.2 \pm 12.6$ \\
\hline LSG & $-8.0 \pm 13.8$ & $-9.4 \pm 14.7$ & $-5.4 \pm 15.8$ & $-10.4 \pm 19.9$ \\
\hline RYGB & $-9.7 \pm 12.7$ & $-8.6 \pm 12.8$ & $-6.7 \pm 13.7$ & $-8.4 \pm 13.8$ \\
\hline$p$ (not adjusted) & $<0.001$ & 0.004 & 0.648 & 0.296 \\
\hline$p$ (adjusted) & $<0.001$ & 0.008 & 0.447 & 0.010 \\
\hline \multicolumn{5}{|l|}{$L D L$} \\
\hline LABG & $-7.6 \pm 33.5$ & $-6.6 \pm 31.6$ & $7.7 \pm 32.1$ & $-13.7 \pm 33.2$ \\
\hline LSG & $-1.1 \pm 32.2$ & $-8.9 \pm 31.6$ & $-12.9 \pm 36.0$ & $-28 \pm 39.8$ \\
\hline RYGB & $-24.1 \pm 30.5$ & $-24.5 \pm 31.1$ & $-25.8 \pm 31.5$ & $-27.3 \pm 29.5$ \\
\hline$p$ (not adjusted) & $<0.001$ & $<0.001$ & $<0.001$ & 0.002 \\
\hline$p$ (adjusted) & $<0.001$ & $<0.001$ & $<0.001$ & 0.001 \\
\hline \multicolumn{5}{|l|}{$\overline{H D L}$} \\
\hline LABG & $5.5 \pm 8.6$ & $6.8 \pm 9.0$ & $5.9 \pm 9.1$ & $6.6 \pm 10.3$ \\
\hline LSG & $7.5 \pm 8.7$ & $9.3 \pm 9.7$ & $9.5 \pm 10.6$ & $8.8 \pm 11.5$ \\
\hline RYGB & $7.0 \pm 11.3$ & $10.6 \pm 11.4$ & $11.3 \pm 12.5$ & $11.6 \pm 11.9$ \\
\hline$p$ (not adjusted) & 0.074 & $<0.001$ & $<0.001$ & 0.002 \\
\hline$p$ (adjusted) & 0.113 & 0.001 & $<0.001$ & 0.014 \\
\hline \multicolumn{5}{|l|}{$T G$} \\
\hline LABG & $-28.4 \pm 65.6$ & $-29.3 \pm 58.4$ & $-30.9 \pm 74.6$ & $-40.6 \pm 68.3$ \\
\hline LSG & $-41.9 \pm 62.5$ & $-41.7 \pm 59.0$ & $-41.5 \pm 61.5$ & $-39.1 \pm 56.5$ \\
\hline RYGB & $-51.9 \pm 80.3$ & $-49.9 \pm 86.0$ & $-51.1 \pm 88.4$ & $-47.4 \pm 59.7$ \\
\hline$p$ (not adjusted) & $<0.001$ & 0.010 & 0.034 & 0.525 \\
\hline$p$ (adjusted) & $<0.001$ & 0.014 & 0.022 & 0.748 \\
\hline \multicolumn{5}{|l|}{ Glycemia } \\
\hline LABG & $-5.1 \pm 24.0$ & $-5.9 \pm 31.1$ & $-5.2 \pm 29.1$ & $-4.6 \pm 39.2$ \\
\hline LSG & $-15.2 \pm 26.7$ & $-13.7 \pm 26.8$ & $-11.0 \pm 24.0$ & $-12.3 \pm 39.2$ \\
\hline RYGB & $-17.8 \pm 32.5$ & $-16.5 \pm 34.3$ & $-15.1 \pm 36.9$ & $-12.9 \pm 29.6$ \\
\hline$p$ (not adjusted) & $<0.001$ & 0.001 & 0.007 & 0.093 \\
\hline$p$ (adjusted) & $<0.001$ & $<0.001$ & $<0.001$ & 0.044 \\
\hline
\end{tabular}


Table 6 (continued)

\begin{tabular}{lcccc}
\hline & Year 1 & Year 2 & Year 3 & Year 4 \\
\hline$A 1 c$ & & & & $-0.4 \pm 0.8$ \\
LABG & $-0.4 \pm 0.7$ & $-0.4 \pm 0.7$ & $-0.4 \pm 0.8$ & $-0.5 \pm 0.6$ \\
LSG & $-0.5 \pm 0.6$ & $-0.5 \pm 0.7$ & $-0.4 \pm 0.6$ & $-0.4 \pm 0.8$ \\
RYGB & $-0.6 \pm 1.0$ & $-0.6 \pm 1.0$ & $-0.5 \pm 0.9$ & 0.767 \\
$p$ (not adjusted) & $<0.001$ & 0.065 & 0.455 & 0.654 \\
$p$ (adjusted) & $<0.001$ & 0.004 & 0.034 & \\
\hline
\end{tabular}

Values are presented as means \pm SD and are adjusted for gender and age. A1c, glycated hemoglobin.

Table 7. Obesity-related comorbidities by years and type of surgery

\begin{tabular}{|c|c|c|c|c|c|}
\hline & Year 0 & Year 1 & Year 2 & Year 3 & Year 4 \\
\hline Total & $n=1,837$ & $n=1,655$ & $n=1,092$ & $n=754$ & $n=473$ \\
\hline \multicolumn{6}{|l|}{ Diabetes } \\
\hline LAGB & 34.7 & 23.6 & 26.2 & 24.9 & 24.2 \\
\hline LSG & 30.2 & 12.7 & 14.1 & 14.4 & 20.0 \\
\hline RYGB & 28.3 & 9.2 & 8.0 & 8.6 & 8.8 \\
\hline$p$ (not adjusted) & 0.114 & $<0.001$ & $<0.001$ & $<0.001$ & $<0.001$ \\
\hline$p$ (adjusted) & 0.491 & $<0.001$ & $<0.001$ & $<0.001$ & 0.005 \\
\hline \multicolumn{6}{|l|}{ Hypertension } \\
\hline LAGB & 66.7 & 49.5 & 53.8 & 53.1 & 47.4 \\
\hline LSG & 56.3 & 36.4 & 32.3 & 41.3 & 34.9 \\
\hline RYGB & 57.2 & 27.0 & 25.5 & 27.9 & 23.1 \\
\hline$p$ (not adjusted) & 0.012 & $<0.001$ & $<0.001$ & $<0.001$ & $<0.001$ \\
\hline$p$ (adjusted) & 0.037 & $<0.001$ & $<0.001$ & $<0.001$ & 0.003 \\
\hline \multicolumn{6}{|l|}{ Dyslipidemia } \\
\hline LAGB & 49.1 & 36.0 & 38.6 & 37.4 & 38.3 \\
\hline LSG & 45.6 & 29.0 & 31.0 & 39.1 & 32.8 \\
\hline RYGB & 40.3 & 13.1 & 10.5 & 11.7 & 10.9 \\
\hline$p$ (not adjusted) & 0.014 & $<0.001$ & $<0.001$ & $<0.001$ & $<0.001$ \\
\hline$p$ (adjusted) & 0.246 & $<0.001$ & $<0.001$ & $<0.001$ & $<0.001$ \\
\hline \multicolumn{6}{|c|}{ Metabolic syndrome } \\
\hline LAGB & 60.6 & 28.0 & 26.0 & 29.4 & 31.6 \\
\hline LSG & 69.2 & 15.4 & 15.3 & 26.2 & 22.9 \\
\hline RYGB & 64.0 & 11.2 & 8.2 & 10.4 & 8.0 \\
\hline$p$ (not adjusted) & 0.196 & $<0.001$ & $<0.001$ & $<0.001$ & $<0.001$ \\
\hline$p$ (adjusted) & 0.055 & $<0.001$ & $<0.001$ & $<0.001$ & 0.001 \\
\hline
\end{tabular}

Values are presented as percents and are adjusted for sex and age.

\section{Impact on Metabolic Parameters}

The decreases in HbA1c were consistent over the first 2 years in RYGB and LSG (Table 6), with reductions of $0.6 \pm 1.0$ and $0.5 \pm 0.6 \%$, respectively, and during all of the years in the LAGB group, with reductions of $0.4 \pm 0.7 \%$. In year 4 , the $\mathrm{HbA} 1 \mathrm{c}$ reduction was similar between groups ( $p=0.654)$. WC and HC reductions were consistently higher in the RYGB group ( $p<$ 
0.001), as was BP reduction; however, SBP was only reduced after year 1 and DBP was only reduced after the first 2 years. LDL reduction was also higher in the RYGB group over 4 years $(p<0.001)$ and TG reduction occurred in the first three years $(p<0.001,0.014$, and 0.022 for 12,24 , and 36 months, respectively). The HDL increase was higher in this same group after the first year ( $p<0.001$ for 24 and 36 months; $p<0.014$ for 48 months).

RYGB (Table 7) was the surgical procedure that caused the highest clinical remission of diabetes ( $p<0.001$ for the first 3 years; $p=0.005$ for year 4$)$, hypertension $(p<0.001$ for the first 3 years; $p=0.003$ for year 4 ), dyslipidemia ( $p<0.001$ for all years), and MetS $(p<0.001$ for all years).

\section{Discussion}

This analysis reinforces the observation that BS is an effective procedure for WL in cases of morbid obesity, resulting in a greater loss during the first year after surgery. Over time, the effectiveness of the procedures is attenuated, especially with LSG. There was an improvement in MetS parameters with all of the techniques, but the improvement was even greater with RYGB.

In this study, a mean EWL of approximately $78 \%$ in the RYGB group was achieved, as well as rates of 73\% in the LSG group and 39\% in the LAGB group at 12 months postoperatively and $72.3,53.3$, and $38 \%$ at 48 months, respectively. A recent study [13], with a similar design but a smaller sample, reported a WL similar to that in our study in patients who were subject to RYGB and LSG regarding TWL and total percent of TWL, but an incidence of less percent of EWL. This was probably because the patients in our study were heavier, and patients with a higher baseline weight have less robust WL as a result of this technique [32].

Regarding the difference between WL with surgical techniques, studies comparing LGS and RYGB show conflicting results, although they usually demonstrate comparable or slightly greater WL with RYGB $[4,5,18,31]$. In our cohort, a significantly greater WL in all parameters (TWL, percent of TWL, and percent of EWL) with RYGB was observed. Significant differences between WC and HC reduction were also observed, which showed a positive effect of RYGB at all times. There is a consensus in most studies regarding the inferiority of the effectiveness of LABG $[6,16]$.

Several studies have demonstrated a significant WL during the first 12 months, followed by a modest one [32]. In our cohort, patients in all groups also registered a clear weight regain over time, mostly in patients in the LSG group. Some studies with longer follow-up periods comparing LSG with RYGB have also suggested that patients who undergo the former technique experience a greater weight regain $[8,11,29,30]$.

Markers of comorbidities in our cohort generally showed a positive effect of RYGB, specifically with significantly improved LDL and HDL for all years. Similarly, previous studies have reported a more beneficial effect on lipid metabolism with RYGB [17], even when matched for baseline BMI and EWL [7]. The proposed mechanisms for these improvements are WL, reduced central obesity, and a change in incretins [22].

SBP decreased similarity with all techniques; however, DBP in the RYGB group decreased more than other groups at 1 and 4 years (with adjustment for sex and age), with the mean percentage of patients labeled as having hypertension being more significantly reduced in the RYGB group. A similar BP reduction had already been reported in previous studies when comparing RYGB and LSG [13], but with some differences, thus favoring RYGB. The majority of studies indicate that RYGB or LSG is superior when compared to LAGB procedures with regard to the resolution of hypertension [23]. However, in our cohort, as the prevalence of hypertension was different among groups, it was difficult to determine the superiority in this aspect. 
Several studies comparing RYGB and LSG with regard to T2DM remission show better results with RYGB $[19,28]$. In our study, RYGB also showed superiority over LSG in glucose metabolism, which was perhaps influenced by a greater WL. However, the results are conflicting, as some studies show similar results between both techniques [25]. Data regarding $\mathrm{HbA1C}$ evolution after surgery must be interpreted with caution, as antidiabetic medications can also influence WL.

Older patients had less WL $(p<0.001)$, except at 48 months $(p=0.102)$. It has been reported that patients older than 45 years lose a smaller amount of excess BMI than younger patients do. The former could suffer from impaired metabolic capacity and a longer duration of comorbidities, which could have influenced these results [9].

The decreases in EWL were more prevalent in women than in men during the first 3 years; however, the reduction of BMI was similar, perhaps due to the fact that women had a lower initial weight. Previous reports have shown conflicting results, with some reporting a slightly greater degree of WL in males [24] and others in females [2], and others reporting no significant differences $[1,24]$. The male sample size was smaller than the female one, which may have influenced the results.

Our data suggests that WL in diabetic patients is inferior to that in nondiabetics - as demonstrated previously [26]. A possible explanation for this may be certain metabolic differences between both groups. Patients with remission of T2DM during follow-up had a greater WL and BMI decrease during the first 3 years of follow-up. We can thus speculate that this association is due to the fact that the remission of diabetes that occurs in patients undergoing this type of surgery is higher in those with the greatest WL - as previously suggested in other studies [27].

Patients with hypertension had less WL at 12 months $(p=0.009)$ and those with MetS had less at 24 months $(p=0.020)$. As far as we know, this is the first study to evaluate the impact of these comorbidities on WL. This divergence may be due to differences in their initial weight, the drugs they used, and other unanalyzed variables. The lack of a difference in WL between groups for some variables over time is probably due to the reduction of the sample size over the years.

The main strength of this study is the large number of subjects, who were consecutively selected at one center and included patients who had undergone 3 different surgical techniques (malabsorptive and restrictive procedures). Furthermore, to our knowledge, this is the first study to focus on the impact of obesity comorbidities on WL, which adds new data to the study of the benefits of different techniques on the various metabolic parameters.

There are some limitations to our study, such as the incomplete data and the retrospective nature of our project. With regard to patients with T2DM, the type of antidiabetic medications before and after surgery was not included in the analysis. In addition, only $25.7 \%$ of the total patients experienced 4 years of follow-up in our study. The patients who remained in this study were the heavier ones, which may have led to underestimation of the results (as most of those who remained in the group had been submitted to the LAGB). Another potential limitation in the interpretation of these results is the inequality of the 3 groups - an example being a higher baseline weight in LSG. Previous analyses have shown that a lower preoperative weight predicts a lower EWL, which can strengthen a superior WL with RYGB relative to LSG.

Regarding research on this matter, our study had a larger population than other series, and it included patients who underwent 3 different surgical techniques (malabsorptive and restrictive procedures), which can lead to some of the above mentioned limitations being compensated for. 
Although the benefits demonstrated by the use of RYGB in resolving comorbidities may have been partly due to its greater WL, our main objective was to describe the impact of different techniques on the various metabolic aspects in a large sample of patients in a center of high differentiation.

Additional studies are needed to provide clearer answers regarding long-term weight maintenance and the resolution of comorbidities after experiencing these 3 procedures.

\section{Conclusion}

RYGB showed the largest percent of WL during 4 years of follow-up and it provided more effective outcomes with regard to obesity-related comorbidities. LSG is also an effective BS; however, it is less effective than the former. The application of a gastric band is less effective and should only be used in very specific situations.

\section{Acknowledgement}

We would like to acknowledge the support of all of the endocrinologists, surgeons, and nutritionists of the Multidisciplinary Group for Surgical Management of Obesity.

\section{Statement of Ethics}

All of the procedures performed in studies involving human participants were in accordance with the ethical standards of the national guidelines.

\section{Disclosure Statement}

The authors declare that they have no conflict of interests.

\section{Funding Sources}

No funding was sought for this study.

\section{Author Contributions}

V.G. collected the data, followed up the patients, and drafted this paper. J.S.N. collected the data, followed up the patients, carried out the statistical analysis, and reviewed this paper. D.S., M.J.F., S.C.O., P.S., J.P., and D.M. collected the data and reviewed this paper. A.V. followed up the patients and reviewed this paper; PF followed up the patients and meticulously reviewed this paper; D.C. meticulously reviewed this paper. The AMTCO Group followed up the patients and all of the authors approved the final version of this paper. 
Guerreiro et al.: WL MetS Remission after BS

\section{References}

1 Baldridge AS, Pacheco JA, Aufox SA, Kim KY, Silverstein JC, Denham W, et al. Factors Associated With LongTerm Weight Loss Following Bariatric Surgery Using 2 Methods for Repeated Measures Analysis. Am J Epidemiol. 2015 Aug;182(3):235-43.

2 Bekheit M, Katri K, Ashour MH, Sgromo B, Abou-ElNagah G, Abdel-Salam WN, et al. Gender influence on longterm weight loss after three bariatric procedures: gastric banding is less effective in males in a retrospective analysis. Surg Endosc. 2014 Aug;28(8):2406-11.

3 Celio AC, Wu Q, Kasten KR, Manwaring ML, Pories WJ, Spaniolas K. Comparative effectiveness of Roux-en-Y gastric bypass and sleeve gastrectomy in super obese patients. Surg Endosc. 2017 Jan;31(1):317-23.

4 Cheung D, Switzer NJ, Gill RS, Shi X, Karmali S. Revisional bariatric surgery following failed primary laparoscopic sleeve gastrectomy: a systematic review. Obes Surg. 2014 Oct;24(10):1757-63.

5 Coblijn UK, Verveld CJ, van Wagensveld BA, Lagarde SM. Laparoscopic Roux-en-Y gastric bypass or laparoscopic sleeve gastrectomy as revisional procedure after adjustable gastric band-a systematic review. Obes Surg. 2013 Nov;23(11):1899-914.

6 Colquitt JL, Pickett K, Loveman E, Frampton GK. Surgery for weight loss in adults. Cochrane Database Syst Rev. 2014 Aug;(8):CD003641.

7 Cunha FM, Oliveira J, Preto J, Saavedra A, Costa MM, Magalhães D, et al. The Effect of Bariatric Surgery Type on Lipid Profile: An Age, Sex, Body Mass Index and Excess Weight Loss Matched Study. Obes Surg. 2016 May; 26(5):1041-7.

8 Dogan K, Gadiot RP, Aarts EO, Betzel B, van Laarhoven CJ, Biter LU, et al. Effectiveness and Safety of Sleeve Gastrectomy, Gastric Bypass, and Adjustable Gastric Banding in Morbidly Obese Patients: a Multicenter, Retrospective, Matched Cohort Study. Obes Surg. 2015 Jul;25(7):1110-8.

9 Frattini F, Rausei S, Ferrari C, Rovera F, Boni L, Dionigi G. Need of standardization in bariatric surgery: is it time to think about? Comment on Contreras J E, Santander C, Court I, Bravo J. Correlation between age and weight loss after bariatric surgery. Obesity Surgery 2013; 23(8):1286-9. Obes Surg. 2014 Nov;24(11):1994.

10 Gloy VL, Briel M, Bhatt DL, Kashyap SR, Schauer PR, Mingrone G, et al. Bariatric surgery versus non-surgical treatment for obesity: a systematic review and meta-analysis of randomised controlled trials. BMJ. 2013 Oct; 347:f5934.

11 Himpens J, Dobbeleir J, Peeters G. Long-term results of laparoscopic sleeve gastrectomy for obesity. Ann Surg. 2010 Aug;252(2):319-24.

12 Kang JH, Le QA. Effectiveness of bariatric surgical procedures: A systematic review and network meta-analysis of randomized controlled trials. Medicine (Baltimore). 2017 Nov;96(46):e8632.

13 Lager CJ, Esfandiari NH, Luo Y, Subauste AR, Kraftson AT, Brown MB, et al. Metabolic Parameters, Weight Loss, and Comorbidities 4 Years After Roux-en-Y Gastric Bypass and Sleeve Gastrectomy. Obes Surg. 2018 Nov; 28(11):3415-23.

14 Lee JH, Nguyen QN, Le QA. Comparative effectiveness of 3 bariatric surgery procedures: Roux-en-Y gastric bypass, laparoscopic adjustable gastric band, and sleeve gastrectomy. Surg Obes Relat Dis. 2016 Jun;12(5): 997-1002.

15 Lee SK, Heo Y, Park JM, Kim YJ, Kim SM, Park J, et al. Roux-en-Y Gastric Bypass vs. Sleeve Gastrectomy vs. Gastric Banding: The First Multicenter Retrospective Comparative Cohort Study in Obese Korean Patients. Yonsei Med J. 2016 Jul;57(4):956-62.

16 Lee WJ, Lee KT, Ser KH, Chen JC, Tsou JJ, Lee YC. Laparoscopic adjustable gastric banding (LAGB) with gastric plication: short-term results and comparison with LAGB alone and sleeve gastrectomy. Surg Obes Relat Dis. 2015 Jan-Feb;11(1):125-30.

17 Li J, Lai D, Wu D. Laparoscopic Roux-en-Y Gastric Bypass Versus Laparoscopic Sleeve Gastrectomy to Treat Morbid Obesity-Related Comorbidities: a Systematic Review and Meta-analysis. Obes Surg. 2016 Feb;26(2): 429-42.

18 Mahawar KK, Graham Y, Carr WR, Jennings N, Schroeder N, Balupuri S, et al. Revisional Roux-en-Y Gastric Bypass and Sleeve Gastrectomy: a Systematic Review of Comparative Outcomes with Respective Primary Procedures. Obes Surg. 2015 Jul;25(7):1271-80.

19 Menguer RK, Weston AC, Schmid H. Evaluation of Metabolic Syndrome in morbidly Obese Patients Submitted to Laparoscopic Bariatric Surgery: Comparison of the Results between Roux-En-Y Gastric Bypass and Sleeve Gastrectomy. Obes Surg. 2017 Jul;27(7):1719-23.

20 Mingrone G, Panunzi S, De Gaetano A, Guidone C, Iaconelli A, Nanni G, et al. Bariatric-metabolic surgery versus conventional medical treatment in obese patients with type 2 diabetes: 5 year follow-up of an open-label, single-centre, randomised controlled trial. Lancet. 2015 Sep;386(9997):964-73.

21 Nassour I, Almandoz JP, Adams-Huet B, Kukreja S, Puzziferri N. Metabolic syndrome remission after Roux-enY gastric bypass or sleeve gastrectomy. Diabetes Metab Syndr Obes. 2017 Sep;10:393-402.

22 Nora M, Guimarães M, Almeida R, Martins P, Gonçalves G, Santos M, et al. Excess body mass index loss predicts metabolic syndrome remission after gastric bypass. Diabetol Metab Syndr. 2014 Jan;6(1):1.

23 Owen JG, Yazdi F, Reisin E. Bariatric Surgery and Hypertension. Am J Hypertens. 2017 Dec;31(1):11-7.

24 Perrone F, Bianciardi E, Benavoli D, Tognoni V, Niolu C, Siracusano A, et al. Gender Influence on Long-Term Weight Loss and Comorbidities After Laparoscopic Sleeve Gastrectomy and Roux-en-Y Gastric Bypass: a Prospective Study With a 5-Year Follow-up. Obes Surg. 2016 Feb;26(2):276-81. 
25 Peterli R, Borbély Y, Kern B, Gass M, Peters T, Thurnheer M, et al. Early results of the Swiss Multicentre Bypass or Sleeve Study (SM-BOSS): a prospective randomized trial comparing laparoscopic sleeve gastrectomy and Roux-en-Y gastric bypass. Ann Surg. 2013 Nov;258(5):690-4.

26 Schauer PR, Burguera B, Ikramuddin S, Cottam D, Gourash W, Hamad G, et al. Effect of laparoscopic Roux-en Y gastric bypass on type 2 diabetes mellitus. Ann Surg. 2003 Oct;238(4):467-84.

27 Schauer PR, Bhatt DL, Kirwan JP, Wolski K, Aminian A, Brethauer SA, et al. Bariatric surgery versus intensive medical therapy for diabetes - 5-year outcomes. N Engl J Med. 2017;376:641-51.

28 Souteiro P, Belo S, Neves JS, Magalhães D, Silva RB, Oliveira SC, et al. Preoperative Beta Cell Function Is Predictive of Diabetes Remission After Bariatric Surgery. Obes Surg. 2017 Feb;27(2):288-94.

29 Van de Laar A, de Caluwé L, Dillemans B. Relative outcome measures for bariatric surgery. Evidence against excess weight loss and excess body mass index loss from a series of laparoscopic Roux-en-Y gastric bypass patients. Obes Surg. 2011 Jun;21(6):763-7.

30 Vidal P, Ramón JM, Goday A, Benaiges D, Trillo L, Parri A, et al. Laparoscopic gastric bypass versus laparoscopic sleeve gastrectomy as a definitive surgical procedure for morbid obesity. Mid-term results. Obes Surg. 2013 Mar;23(3):292-9.

31 Wang MC, Guo XH, Zhang YW, Zhang YL, Zhang HH, Zhang YC. Laparoscopic Roux-en-Y gastric bypass versus sleeve gastrectomy for obese patients with Type 2 diabetes: a meta-analysis of randomized controlled trials. Am Surg. 2015 Feb;81(2):166-71.

32 Wolfe BM, Kvach E, Eckel RH. Treatment of obesity: weight loss and bariatric surgery. Circ Res. 2016 May 27; 118(11):1844-55. 